Núm. 25, julio-diciembre 2011

\title{
PRESUPUESTOS INDISPENSABLES PARA PROCURAR LA COMPLEMENTARIEDAD DE LAS INSTITUCIONES DEMOCRÁTICAS REPRESENTATIVAS CON EL REFERÉNDUM EN LOS PROCESOS DE REFORMA CONSTITUCIONAL
}

\author{
MANDATORY BUDGETS TO PROCURE \\ THE COMPLEMENTARITY OF REPRESENTATIVE \\ DEMOCRATIC INSTITUTIONS WITH THE REFERENDUM \\ ON CONSTITUTIONAL REFORM PROCESSES
}

\section{Javier PATIÑO CAMARENA*}

RESUMEN: Si la Constitución, además de ser la ley suprema del ordenamiento jurídico positivo, es la ley que delinea el rostro político de la nación, la ley que crea al Estado, la ley que organiza al gobierno y en la cual los ciudadanos han precisado los términos del contrato social con apego al cual han decidido vivir en sociedad, si la constitución tiene esta significación tan rica y variada, ¿cuáles son los presupuestos mínimos indispensables que se deben de observar en todo proceso de reformas a la Constitución? Si lo propio y característico de un Estado democrático de derecho es que los poderes constituidos no puedan en ningún caso reformar a la constitución que los crea y regula, ¿cuáles son los presupuestos mínimos indispensables para evitar este peligro?

Palabras clave: Constitución, reforma constitucional, referéndum
ABSTRACT: If the Constitution, besides being the supreme law of positive law is the law that outlines the political face of the nation, the law creating the State, the law that organizes the government and in which citizens have defined the terms attachment of the social contract which have chosen to live in society, if the constitution has this rich and varied meaning, what are the minimum essential budgets to be observed in any process of reforms to the Constitution?

If you own characteristic of a democratic state of law is that the powers that may in no case to reform the constitution that creates and regulates, what are the minimum budget required to avoid this danger?

Descriptors: Constitution, constitutional amendment, referendum.

*Investigador de tiempo completo en el Instituto de Investigaciones Jurídicas de la UNAM. 


\section{EXIGENCIAS DE LA DEMOCRACIA EN NUESTROS DÍAS}

Las democracias representativas se estructuran a partir del principio de que la titularidad de la soberanía le corresponde al pueblo, el cual, por razones prácticas delega el ejercicio de algunas de sus atribuciones soberanas en las personas que elige como sus representantes y las cuales sólo pueden actuar dentro de los límites que la voluntad general les ha precisado en la Constitución. Pero el pueblo ni delega la titularidad de su soberanía, ni delega el ejercicio de todas sus atribuciones soberanas, sino que tan solo delega el ejercicio de unas cuantas, y el ejercicio de aquellas atribuciones que delega, no lo hace de manera irrevocable, de tal manera que el pueblo permanece en calidad de soberano y en cualquier momento puede revocar el ejercicio de aquellas atribuciones que haya delegado y asumir su ejercicio directo.

Pero para estructurar a una democracia representativa, no basta con afirmar y reafirmar que la soberanía radica original y esencialmente en el pueblo, sino que además se requiere configurar un sistema representativo, el cual supone, como mínimo, la creación de un sistema de partidos políticos que haga posible que la divergencia de opiniones, propia y característica de la democracia, se pueda reducir a proporciones racionales que permitan organizar la vida política, económica y social del país y de igual forma supone el que con base en el sufragio universal se organicen consultas electorales a intervalos de relativa frecuencia, que permitan conocer la opinión de los ciudadanos acerca de quiénes deben ser sus representantes, así como el programa político con apego al cual desean que se gobierne al país.

Ahora bien, los regímenes democráticos más avanzados, ya no se limitan a afirmar y reafirmar el principio de la soberanía del pueblo, ni a estructurar y mejorar al sistema representativo, sino que además buscan configurar mecanismos que aseguren que el pueblo ejerza su soberanía no solo eligiendo a sus representantes, sino también aprobando en definitiva las decisiones más importantes y trascendentes que se relacionan con los términos del contrato social precisado en la Constitución. Por ello un gran número de tratadistas ven al referéndum como la figura emblemática de la democracia semidirecta que permite tener un auténtico gobierno del pueblo, lo que ha dado como resultado que en los foros académicos se hayan incrementado notablemente los pronunciamientos en favor de que se adopte el referéndum, al menos en relación con las reformas constitucionales. 


\section{El Poder CONSTITUyente}

De conformidad con la doctrina constitucionalista, la titularidad del Poder Constituyente le corresponde al titular del poder soberano, el cual para el pensamiento democrático no es otro que el pueblo, es decir, la voluntad general, la mayoría, razón por la cual se puede decir que el Poder Constituyente viene a significarse por cuanto es la primera y más importante manifestación de la soberanía.

La existencia del Poder Constituyente obedece a la consideración de que las condiciones de vida de la época contemporánea imposibilitan el que se reúnan todos los ciudadanos en un mismo lugar y fecha para precisar los términos de la "voluntad común" que debe regular la vida en sociedad, razón por la cual el pueblo elige a los integrantes de un Poder Constituyente al que se le responsabiliza de elaborar una Constitución, en la que se precisen los términos en que el pueblo soberano delega el ejercicio de algunas de sus atribuciones en los poderes constituidos, los cuales en ningún caso y por ningún motivo pueden modificar los términos de la voluntad general precisados en la Constitución.

De aquí que se afirme que la diferenciación entre el Poder Constituyente y los poderes constituidos es un rasgo esencial de todo Estado social y democrático de derecho, y ello explica que dicha distinción haya sido y sea combatida por los partidarios de cualquier tipo de absolutismo.

En este orden de ideas cabe precisar que el Poder Constituyente es un poder originario, de definición política al que le corresponde la función de constituir políticamente a la nación, y plasmar en una Constitución la "idea de derecho" con apego a la cual la voluntad general, la mayoría, quiere que se regule la vida en sociedad, en tanto que los poderes constituidos son poderes creados, poderes a los que les corresponde gobernar con apego a los principios, reglas y procedimientos que el poder constituyente ha precisado en la Constitución.

La diferenciación entre el Poder Constituyente y los poderes constituidos, ha dado como resultado la configuración del principio de supremacía constitucional, eje fundamental en el propósito de construir un Estado social y democrático de derecho, en el que resulta fundamental importancia el que a través de diversos mecanismos se asegure que los poderes constituidos ejerzan el poder con escrupuloso apego a lo dispuesto por la Constitución, ya que es de esta forma como se asegura que el ejercicio del poder se encuentre sometido y regulado por el derecho. Es por ello que se afirma que 
la Constitución es una ley fundamental, ya que los cuerpos políticos que existen por ella, en ningún caso pueden modificarla, siendo que por el contrario en todo momento tienen que ajustar su actuación a los principios y procedimientos establecidos en la Constitución

El Poder Constituyente se puede materializar o concretizar a través de la integración de asambleas constituyentes determinativas o bien a través de asambleas constituyentes proyectistas. Se estará en presencia de la primera modalidad cuando el pueblo elige representantes constituyentes a los que se les confiere el mandato de elaborar un proyecto de Constitución, el cual adquirirá este carácter si es aprobado por la mayoría de los integrantes de dicha asamblea.

En cambio se estará en presencia de una Asamblea Constituyente proyectista, en los caso en que el pueblo elige representantes constituyentes a los que se les confiera el mandato de elaborar un proyecto de Constitución, en el entendido de que si el proyecto en cuestión es aprobado por la mayoría de los integrantes de dicha asamblea, no por ello se convertirá en la Constitución, sino que dicha aprobación es la condición indispensable para que el proyecto en cuestión sea sometido a la aprobación definitiva del pueblo por la vía del referéndum. En este caso cobra sentido la afirmación de que el Poder Constituyente es el pueblo actuando, que aprueba su Constitución.

\section{LA CONSTITUCIÓN}

La obra del Poder Constituyente, ya sea que adopte para su organización la modalidad de una Asamblea Constituyente proyectista o determinativa, se plasma y concretiza en la elaboración de una Constitución, la cual viene a significarse por ser la ley por medio de la cual el pueblo delinea el rostro político de la nación.

De aquí que la primera preocupación del constitucionalismo haya consistido en explicar el papel protagónico que le corresponde al pueblo en su elaboración (Rousseau, Paine, Sieyes, Mounier, Rossi), y hayan puesto especial énfasis en señalar que una Constitución es el acto por virtud del cual un pueblo se da un gobierno, y que un gobierno sin Constitución es un poder sin derecho; con posterioridad, cuando quedó claro que las Constituciones son obra del pueblo, se procuró desentrañar su esencia (Lassalle, Schmitt), más tarde algunos autores como Kelsen centraron su atención en el nivel jerárquico que tiene la Constitución en el marco de un sistema 
jurídico positivo y las implicaciones que ello tiene en la configuración de todo sistema de derecho positivo, en tanto que otros autores, como Maurice Haurieau, le confirieron especial atención a la finalidad que persigue todo régimen constitucional.

Los elementos que a través del tiempo se han tomado en cuenta con objeto de conceptualizar a la Constitución (el papel protagónico del pueblo, su naturaleza o esencia, el nivel jerárquico que tiene dentro del derecho positivo y su finalidad), pueden ser amalgamados con el propósito de formular una conceptualización integral conforme a la cual la Constitución es la ley suprema de todo orden jurídico que expresa en forma normativa el papel que guardan los factores reales de poder que existen en una colectividad y en la cual el pueblo soberano consagra las decisiones políticas y jurídicas fundamentales con apego a las cuales se debe organizar y ejercer racionalmente al poder (Estado y gobierno ) a fin de procurar un equilibrio entre orden y libertad o, si se prefiere, entre las fuerzas de resistencia al cambio y las fuerzas transformadoras.

En este orden de ideas, se puede decir que la alta significación que tiene la Constitución deriva del hecho de que a través de ésta ley suprema se delinea el perfil político de la nación, se sientan las bases organizativas del Estado, las bases funcionales del gobierno, se precisan los términos del contrato social con apego al cual los miembros de una comunidad han decidido vivir en sociedad y consecuentemente se delinea la "Idea de derecho" que debe regir la vida en sociedad y se configuran las garantías constitucionales, o derechos públicos subjetivos fundamentales que precisan las finalidades individuales, políticas y sociales que se pretenden alcanzar.

\section{EL PODER CONSTITUCIONAL REFORMADOR}

Las Constituciones tienen que ser estables a efecto de que los integrantes de un cuerpo social conozcan sus disposiciones y las graven en sus corazones para de esta suerte estar en condiciones de exigir su invariable respeto y cumplimiento.

Pero una Constitución meramente estable, que permanezca sorda a los cambios que reclama la vida en sociedad como necesarios, al poco tiempo sería una pieza de arqueología jurídica, una antigualla, un cadáver in sepulto, de poca o nula utilidad para regular con eficacia la vida en sociedad, en tanto que una Constitución que sea objeto de frecuentes cambios, atentará en contra del principio de seguridad jurídica, ya que ello le im- 
pedirá al cuerpo social saber a qué orden atenerse. De aquí que se pueda decir que la salud de un régimen constitucional depende del acierto con el que se logre un equilibrio entre estabilidad y cambio constitucional.

Así, con objeto de alcanzar este fin y poder armonizar en forma continua las formas políticas y jurídicas contenidas en la Constitución con los cambios que la realidad social exija como necesarios, sin tener que recurrir al expediente del poder Constituyente Originario, (ya que éste por su propia naturaleza solo emerge en los momentos de profunda crisis política) la doctrina constitucionalista ha ideado diversos mecanismos que tienen como común denominador el que evitan que los poderes constituidos puedan reformar a la Constitución, ya que el principio de supremacía constitucional, piedra de toque del llamado Estado de derecho o del Estado social y democrático de derecho, parte del reconocimiento de que los poderes constituidos en ningún caso y por ningún motivo pueden modificar a la Constitución, porque si tal cosa pudieran hacer, el ejercicio del poder no estaría regulado por el derecho, por la Constitución, sino que en este caso la Constitución estaría sometida al capricho de los poderes políticos.

De aquí que se pueda afirmar que el propósito medular del Estado de derecho y las implicaciones del principio de supremacía constitucional condujeron a la doctrina constitucionalista al convencimiento de que para poder reformar la Constitución sin tener que recurrir al expediente del Poder Constituyente originario, se le tiene que confiar dicha responsabilidad a un poder que tenga una naturaleza intermedia entre los poderes constituidos y el Poder Constituyente originario.

En este orden de ideas se puede decir que el poder al que se faculta para reformar a la Constitución, se asemeja al Poder Constituyente, por cuanto el ejercicio de la función de ambos da como resultado la elaboración de normas constitucionales, pero se diferencian en cuanto a su origen, ya que el Poder Constituyente es un poder originario, es el representante directo e inmediato del pueblo, es el pueblo actuando que se da su Constitución, en tanto que el Poder que reforma a la Constitución tiene su origen en la Constitución elaborada por el Poder Constituyente.

Por otra parte, el Poder que puede reformar a la Constitución se asemeja a los poderes constituidos por su origen, ya que en uno y otro caso su existencia se encuentra prevista en la Constitución elaborada por el Poder Constituyente, pero se diferencia de ellos por su función, ya que los poderes constituidos tiene como función la de gobernar con apego a los principios y procedimientos establecidos en la Constitución, en tanto que la función 
del poder constitucional reformador es la de introducirle a la Constitución los cambios que la realidad social reclame como necesarios

Al mecanismo o al poder legitimado para introducirle cambios a la voluntad general expresada en la Constitución, por lo general se le denomina Poder Constituyente permanente, pero ésta denominación no resulta satisfactoria, en primer término, porque parece conferirle un peso, rango y jerarquía mayor a la del Poder Constituyente propiamente dicho, que es un poder originario y el cual por su naturaleza tiene una existencia transitoria y fugaz; en segundo lugar, dicha denominación es criticable por inexacta, ya que dicho poder no tiene una existencia permanente, toda vez que su existencia es, en todo caso, intermitente, puesto que su presencia se concretiza solo cuando se pone en movimiento el mecanismo para reformar la Constitución, lo que puede tener lugar una o varias veces durante un gobierno o no actualizarse durante dicho término y, por último, la denominación "Poder Constituyente permanente" resulta criticable por cuanto choca frontalmente con el principio de "seguridad jurídica", que resulta esencial en el Estado de derecho, ya que en caso de que existiera realmente un Poder Constituyente permanente, ello le imposibilitaría a la ciudadanía saber a qué atenerse, toda vez que la Constitución podría modificarse cotidianamente.

Otra denominación que se utiliza con frecuencia, para denominar a este "poder" es la de "Poder Revisor", pero ésta denominación tampoco resulta satisfactoria, toda vez que su enunciado traduce la idea de un poder "corrector" de las decisiones adoptadas por el Poder Constituyente originario, lo cual no corresponde a la realidad, ya que la actuación del llamado "Poder Revisor" no tiene por objeto "revisar" la obra del Poder Constituyente, sino que la razón de ser de su existencia deriva de la necesidad de introducirle a la Constitución los cambios que la realidad social exija como necesarios. Pero además la denominación poder revisor induce a la confusión, ya que da lugar a que se piense que es un poder superior al Poder Constituyente, ya que quien revisa se coloca, por efectos de su actuación, en un plano superior respecto de quien es objeto de la revisión, lo que en el caso de reformas a la Constitución no corresponde a la realidad, ya que el Poder Constituyente es la expresión por excelencia del poder soberano y sus integrantes fueron electos con el propósito expreso de constituir políticamente a la nación, en tanto que el llamado Poder Revisor fue creado por el Poder Constituyente al elaborar la Constitución; por último, se puede decir que el término "poder revisor" resulta desbordado en sus términos en los casos en los que a través de su actuación se le adi- 
ciona un nuevo enunciado jurídico a la Constitución, pues las adiciones, por su propia naturaleza, no pueden ser consideradas como revisiones y en caso de que así se hiciera ello constituiría un despropósito.

Con base en las consideraciones anteriores se propone que al mecanismo ideado para introducirle cambios a la Constitución, se le denomine "Poder Constitucional Reformador", ya que de esta forma se explicita tanto su origen, que es la Constitución, como la función que le es propia, la cual consiste en reformar la Constitución con objeto de recoger los cambios que se estimen necesarios para armonizar en forma continua los principios políticos y jurídicos contenidos en la Constitución con las formas reales de vida.

En este estado de cosas, para regular la organización y el funcionamiento del poder constitucional reformador, se han ideado dos grandes modelos que atienden a la forma como se organiza al Estado. A dichos modelos la doctrina los conoce como el modelo central o francés y el modelo federal o americano, modelos que tienen como denominador común el que a través de ellos se busca evitar que la Constitución pueda ser reformada por alguno de los poderes constituidos, para de esta suerte preservar al principio de supremacía constitucional.

El modelo central parte de la consideración de que en un país organizado en forma centralizada, solo existe un poder legislativo, un poder de definición política, por lo que para modificar la Constitución se requiere de un mecanismo que se integre a través de la actividad concertada de dos o más legislaturas que se sucedan en el tiempo, disponiéndose, en su fórmula más sencilla, que a la legislatura en turno le corresponde precisar los términos de la propuesta de reformas que se quiere, en tanto que a la legislatura siguiente le corresponderá aprobar o rechazar el proyecto de reformas propuesto por la legislatura precedente. De esta suerte, la legislatura que propone los términos de una reforma no resuelve si se aprueba o no la misma y la legislatura que resuelve sobre la aceptación o el rechazo de la reforma propuesta, no tiene intervención alguna en su formulación, consecuentemente quien propone no resuelve, y quien resuelve no propone, por lo que a través de la actividad concertada de dos legislaturas que se suceden a través del tiempo se evita que la Constitución sea reformada por alguno de los poderes constituidos, ya que ello contraría la esencia de todo Estado de derecho. Cabe destacar que en este sistema entre la legislatura que propone y la legislatura que resuelve necesariamente se deben de celebrar elecciones y en las campañas respectivas por su importancia deben de tener un peso específico la propuesta de reforma constitucional 
en turno, por lo que los candidatos necesariamente deben de tomar partido en favor o en contra de la propuesta de reforma en cuestión y en función de ello los ciudadanos en la jornada electoral respectiva, deberían votar por aquellos candidatos con cuya postura en torno a la reforma propuesta estén de acuerdo.

El modelo federal parte de la consideración de que El Estado federal es un estado formado por varios estados que cuenta, por lo mismo, con varios centros de impulso o de definición política. Se trata de una forma de organización política que se sitúa, por así decirlo, a la mitad del camino entre el Estado central o unitario y la Confederación de Estados. De aquí que la Constitución de un estado federal, además de la alta significación que tiene toda Constitución, tema al que ya se ha hecho referencia, tiene una significación adicional, por cuanto en ella se precisan los términos del "pacto federal", del reparto de competencias entre la Federación y las entidades federativas, reparto que tiene por objeto conciliar "unidad y diversidad". Por todo ello, en el diseño del mecanismo de reformas a la Constitución de un Estado federal reviste particular importancia que tengan participación tanto la Federación como las entidades federativas, razón por la cual el poder constitucional reformador en un Estado federal se integra a través de la actividad concertada entre la legislatura federal y las legislaturas estatales, que al actuar de manera concertada integran un nuevo poder al que en este ensayo se ha propuesto denominar poder constitucional reformador, para de esta forma clarificar su origen, su función y su naturaleza, ya que con esta denominación se clarifica que el poder al que se le responsabiliza de reformar a la Constitución tiene una naturaleza claramente diferenciada de los poderes constituidos (Poder Legislativo federal y poderes legislativos estatales), los cuales, precisamente por tener este carácter, no pueden reformar a la ley que los crea y regula.

\section{EL DERECHO AL VOTO Y EL REFERÉNDUM EN RELACIÓN CON EL PROCESO DE REFORMA CONSTITUCIONAL}

De los derechos políticos el más importante es el derecho al voto, ya que a través del mismo los ciudadanos eligen no solo a sus representantes políticos, sino también a un programa político con apego al cual desea que se gobierne al país y además, algo que frecuentemente se soslaya, a través del voto se elige a las personas que integrarán órganos ( en el caso, las cámaras del Congreso 
de la Unión y las legislaturas de los estados), que eventualmente al actuar de manera concertada en los términos que precisa la ley suprema, configurarán el mecanismo por medio del cual se puede reformar a la Constitución, atribución que tendrán sin que en el momento de su elección, ni ellos ni el cuerpo ciudadano, conozcan el sentido en el que se quiere reformar la Constitución, por lo que ninguna consideración de este tipo gravita en su elección.

Ahora bien, los regímenes democráticos más avanzados, ya no se limitan a organizar el ejercicio del derecho al voto, sino que además procuran configurar mecanismos que aseguren que el pueblo ejerza su soberanía no solo eligiendo a sus representantes, sino también aprobando en definitiva las decisiones más importantes y trascendentes que se relacionan con los términos del contrato social precisado en la Constitución.

Las consideraciones anteriores han dado como resultado que en los foros académicos en los últimos años haya aumentado considerablemente los pronunciamientos en favor de que se adopte y regule al referéndum en relación con los procesos de reforma constitucional, ya que ven en él referéndum el mecanismo idóneo para asegurar que efectivamente el pueblo participe en forma activa en la toma de decisiones relacionadas con las determinaciones constitucionales, pero teniendo buen cuidado de que su reconocimiento se haga en términos tales que efectivamente resulte posible su puesta en práctica y no solo con un propósito retórico que le permita a sus promotores presentarse como un país de avanzada, pero de escasa o nula posibilidad práctica.

En atención al momento en el que opera el referéndum, se habla o bien de un referéndum de consultación, o bien de un referéndum de ratificación. La primera hipótesis se da cuando se le pide al pueblo su parecer antes de elaborar una iniciativa legislativa sobre un tema determinado y en caso de que el resultado sea afirmativo los órganos competentes deben proceder a su elaboración, y en caso de que el resultado sea negativo se debe desechar o abandonar el proyecto legislativo en cuestión. En cambio, por medio del referéndum de ratificación se faculta al pueblo para que apruebe en definitiva ciertas proposiciones de leyes ordinarias o constitucionales que hayan sido elaboradas por los órganos competentes.

Con base en las consideraciones anteriores se puede decir que en tanto que el referéndum de consultación tiene lugar al inicio de un proceso legislativo, el referéndum de ratificación tiene lugar al final del proceso legislativo correspondiente, ya que el pueblo tiene la última 
palabra en relación con un proyecto de ley elaborado por los órganos competentes, proyecto al que aprueba o rechaza en forma definitiva.

En los estudios especializados sobe la materia, frecuentemente se cita como ejemplos de experiencias referendarias positivas y exitosas, a las que tuvieron lugar en España y Chile, la primera tuvo lugar el 6 de diciembre de 1978, cuando después de la muerte del caudillo Francisco Franco se elaboró un proyecto de Constitución que se sometió a la aprobación definitiva del pueblo a través de un referéndum, y el cual fue aprobado por $87.87 \%$ de los electores, en tanto que la experiencia chilena que se cita con frecuencia es la que tuvo lugar el 5 de octubre de 1988, cuando se le preguntó al electorado si quería que continuara en el poder Augusto Pinochet, ocasión en la cual el pueblo chileno se pronunció en favor de que se celebraran elecciones y consecuentemente en contra de la permanencia de Pinochet en el poder.

Cabe aclarar que frecuentemente se utilizan los términos referéndum y plebiscito como sinónimos, no obstante que entre una y otra institución existen notables diferencias. En efecto, en tanto que a través del referéndum se faculta al pueblo para aceptar o rechazar en definitiva un proyecto de ley formulado por los órganos competentes, el plebiscito es el voto de confianza que le otorga el pueblo a una persona para que continúe en el poder. La doctrina francesa cita como casos típicos de plebiscitos, la consulta que se hizo al pueblo para saber si quería la herencia de la dignidad imperial para la descendencia directa, natural, legítima y adoptiva de Napoleón Bonaparte (mayo de 1804) y para determinar si se quería investir a Luis Napoleón del poder imperial (noviembre de 1852).

La confusión que se da entre referéndum y plebiscito, obedece a que la frontera entre una y otra forma de consulta popular no siempre es fácil de precisar, ya que las consultas populares pueden comprender dosis variables de elementos plebiscitarios y referendarios, tal como sucedió durante la $\mathrm{V}$ República Francesa, período durante el cual el presidente Charles de Gaulle acostumbró solicitarle al pueblo que le confiriera un voto de confianza a través de la aprobación que hiciera por la vía del referéndum de una propuesta legislativa que se le sometía a su consideración, resultando oportuno aclarar que se trata de dos cosas distintas, ya que se puede estar en desacuerdo con una propuesta legislativa, sin por ello perder la confianza en el gobierno.

De los estudios que se han elaborado en torno de esta institución, se desprende que el referéndum puede servir tanto como freno para proteger al cuerpo social de propuestas de reforma que impliquen cambios tras- 
cendentes a los términos esenciales del pacto o contrato social plasmado en la Constitución, como para apoyar e impulsar una "nueva idea del derecho" que responda de mejor manera a los requerimientos que demande la realidad social.

Cabe aclarar que el referéndum también ha sido utilizado por gobernantes de temperamento dictatorial (Napoleón III, Hitler, Mussolini y Ceauşescu) que encontraron que el empleo de esta figura jurídica permite manipular a la opinión pública, ya sea por los términos en que se formule la consulta o pregunta, ya sea por la posibilidad que ofrece para alcanzar, a partir de datos o visiones parciales, un apoyo global a una determinada política, o bien, por cuanto el empleo del referéndum le ofrece al gobernante la posibilidad de eludir los mecanismos de control que puede ejercer la oposición en el seno del Congreso.

Con base en las consideraciones anteriores, se puede concluir que el empleo del referéndum puede permitir tanto la consolidación de regímenes democráticos como el fortalecimiento de otros de talante autocrático, por lo que resulta claro que las ventajas que puede presentar el empleo del referéndum dependen no solo de sus bondades intrínsecas, sino que sus resultados se encuentran condicionados por otros factores, como el grado de desarrollo alcanzado por el país que se propone hacer uso de este instrumento, el nivel educativo de la población del país en cuestión (la calidad de un régimen democrático, depende de la calidad de los demócratas que lo practiquen) y la consistencia que registren sus instituciones democráticas representativas.

En relación con este tema, Michelangelo Bovero, en su libro Los desafíos actuales de la democracia (IFE 1995), alerta sobre el hecho de que "la calidad de un régimen democrático no mejora por simplificar el proceso de toma de decisiones, sino que con frecuencia para mejorarla se requiera agregarle varios mecanismos correctivos, de control y de garantía". El referéndum no es la panacea democrática. Como sociedad de ciudadanos, el régimen puede refrescarse con la participación directa de los electores para resolver cuestiones trascendentes de la vida política. Pero, como régimen de moderaciones, debe ser cauteloso frente a su innegable potencial autocrático". 


\section{PRINCIPALES INTERROGANTES QUE SE PLANTEAN}

EN RELACIÓN CON EL MECANISMO DE REFORMA A LA CONSTITUCIÓN

\section{Y AL REFERÉNDUM ILUSTRADAS MEDIANTE EL CASO DE MÉXICO}

En el momento presente en el que, de conformidad con el Informe del PNUD del 2003 se observa que muchas de las autoridades políticas del continente se están viendo rebasadas en la conducción de los procesos políticos por los poderes fácticos, el reconocimiento de la figura del referéndum puede ayudar a insuflarle nuevo aliento a la democracia.

Pero para que ello sea así, se debe de tener buen cuidado de que el reconocimiento del referéndum no se haga en detrimento de las instituciones características de las democracias representativas, pues en tal caso ello puede significar un paso hacia atrás que de cómo resultado la consolidación de gobiernos autocráticos que por esta vía legitimen su gobierno.

Es por ello que en el presente ensayo se ha puesto especial énfasis en señalar que las ventajas que puede presentar el empleo del referéndum dependen no solo de sus bondades intrínsecas, sino también se encuentran condicionadas por otros factores, tales como el grado de desarrollo alcanzado por el país que se propone hacer uso de este instrumento, el nivel de instrucción de su población y la consistencia real que registren sus instituciones democráticas.

En favor de que se den pasos conducentes para el reconocimiento del referéndum en los procesos de reforma constitucional, se puede decir que dicha medida se inscribe dentro de la perspectiva de enriquecer a la democracia; que quienes hoy se oponen a la adopción del referéndum esgrimen las mismas razones que emplearon en el pasado quienes combatieron la adopción de la elección directa y del sufragio universal, por considerar unas veces que el pueblo aún no estaba preparado para ello y otras por considerar que se le debía de conferir una atención prioritaria a otros temas que resultaban más urgentes. Sin embargo, la historia acredita que las naciones que avanzaron y se modernizaron fueron las que decidieron adoptar el sistema de elección directa y el sufragio universal.

También se puede decir que puesto que el referéndum ya es una realidad en las democracias occidentales más avanzadas, resulta lógico y deseable que se den los pasos necesarios para que también lo sea en nuestro medio, sobre todo si se parte de la consideración de que el referéndum solo será una realidad en México, si desde hoy se logra su reconocimiento y se diseñan responsablemente los supuestos constitucionales necesarios para su ejercicio. 
Pero el reconocimiento del referéndum se debe realizar en términos consistentes y no a través de meras fórmulas declarativas que permitan presumir de que se cuenta con instituciones de democracia semidirecta, pero teniendo buen cuidado de dificultar su funcionamiento a través del empleo de fórmulas unas veces "confusas" y otras veces "difusas", como sucedido en 1857, 1977 y 1996 cuando la propuesta de su adopción no se acompañó del andamiaje jurídico idóneo para asegurar su buen funcionamiento, Consecuentemente, si en el presente se quiere reconocer al referéndum se debe de trascender los enfoques erróneos, limitados y parciales que se dieron en el pasado.

Consecuentemente el dilema no radica en decidir si se le debe conferir una atención prioritaria a los problemas fundamentales que plantea la democracia representativa, lo que en los hechos equivaldría a diferir ad infinitum el empleo del referéndum o, si por el contrario, si se le debe conferir una atención preferente al referéndum en detrimento de la atención que se le debe conferir a los problemas torales que plantea la democracia representativa, ya que ello puede dar como resultado el que su empleo sirva para eludir los sistemas de control que corren a cargo del Congreso, sino que, por el contrario, a todos estos planteamientos se les debe alentar a efecto de configurar con ellos la próxima agenda de la Reforma de Estado que cada vez resulta más necesaria.

Con apego a este planteamiento, se considera que la propuesta de someter a referéndum las propuestas de reforma constitucional, se debe ver acompañada con una serie de medidas que tiendan a resolver diversos problemas por demás trascendentes que plantean los modelos de reforma constitucional existentes y los cuales se procurará ilustrar a continuación a través del análisis del mecanismo de reforma constitucional vigente en México.

Con este fin cabe recordar que el artículo 135 de la Constitución Mexicana vigente estructura al Poder Constitucional reformador por medio de la actividad concertada del Congreso de la Unión y de las Legislaturas de los Estados, en los siguientes términos:

Artículo 135. La presente Constitución puede ser adicionada o reformada. Para que las adiciones o reformas lleguen a ser parte de la misma se requiere que el Congreso de la Unión, por el voto de las dos terceras partes de los individuos presentes, acuerde las reformas o adiciones y que éstas sean aprobadas por la mayoría de las Legislaturas de los Estados. El Congreso de 
la Unión hará el cómputo de los votos de las legislaturas y la declaración de haber sido aprobadas las adiciones o reformas.

Este mecanismo de reforma constitucional en apariencia claro y sencillo plantea en realidad múltiples interrogantes trascendentes, si bien en esta ocasión, por razones de espacio y tiempo, tan sólo me ocuparé de media docena de ellas.

¿Quiénes pueden presentar iniciativas de reforma a la Constitución federal?

Sorprende el que en una materia tan importante y trascendente como es la determinación de quienes pueden proponer reformas a los términos de la "voluntad general" expresada en la Constitución, no figure una determinación expresa sobre la materia, como lo hace la Constitución de los Estados Unidos.

En vista de ello, la doctrina constitucional mexicana ha resuelto que las entidades o instancias que tienen facultad para presentar iniciativas de leyes federales, también tienen la facultad para presentar iniciativas de reforma constitucional. Si bien esta postura presenta la ventaja de "cobijar" la práctica que durante 93 años se ha observado en esta materia, no resulta del todo consistente, ya que no es cierto, como algunos han dicho, que esta solución sea producto de la aplicación del principio general de que "quien puede lo más, puede lo menos", ya que en todo caso daría lugar a un nuevo principio conforme al cual habría que reconocer que "quien puede lo menos puede lo más", toda vez que resulta incontrovertible que las leyes federales tienen una jerarquía inferior a la Constitución y por lo mismo no tiene la misma relevancia, ni trascendencia modificar a ésta que a aquéllas. Por eso se considera que esta materia debe ser objeto de una atención preferente en el momento en el que se planté la oportunidad de reformar el mecanismo de reformas a la Constitución.

2. La exigencia constitucional de que toda iniciativa de adición o reforma a la Constitución debe ser aprobada, en primer término, por las $2 / 3$ partes de los miembros presentes del Congreso de la Unión, plantea la interrogante de determinar la manera como debe sesionar dicho cuerpo colegiado, ¿en forma conjunta o separada? Es decir, se requiere determinar si las cámaras que integran al Congreso de la Unión, al ocuparse de una reforma constitucional, deben sesionar en asamblea única, o si deben de sesionar en forma separada, sucesiva, e independiente, como lo hacen cuando se ocupan de iniciativas de leyes federales. 
El argumento de mayor peso que esgrimen los partidarios de que las cámaras sesiones en forma separada al ocuparse de propuestas de reforma a la Constitución, lo hacen consistir en que el análisis de los 189 procesos de reforma a la Constitución, que han tenido lugar desde 1921 hasta septiembre del 2010, pone de manifiesto que en todos los casos las cámaras sesionaron en forma separada y sucesiva, en tanto que los críticos de esta postura consideran que la repetición de una práctica irregular no la convalida.

Al margen de las consideraciones anteriores, se puede decir que el hecho de que las cámaras hayan sesionado en el pasado en forma separada y sucesiva en los procesos de reforma a la Constitución, no es obstáculo para que llegado el momento en el que se considere políticamente conveniente reformar el mecanismo de reformas a la Constitución, los actores políticos resuelvan que por la importancia, relevancia y trascendencia que tiene todo proceso de reforma constitucional, en el futuro las cámaras que integran al Congreso de la Unión tengan que sesionar en forma conjunta, en Asamblea única, como lo hace en Francia el Congreso cuando sesiona en el Palacio de Versalles con objeto de resolver lo conducente en relación con una propuesta de reforma a la Constitución.

3. La disposición que establece que las reformas a la Constitución deben ser aprobadas, en primer término por las $2 / 3$ partes de los miembros del Congreso de la Unión presentes ¿realmente configura un quórum calificado que asegura que la reforma aprobada cuenta con un amplio consenso en favor de la misma?

Frecuentemente la doctrina recomienda que en materia de reformas constitucionales no se admitan sino las que aparezcan indispensables y que sean susceptibles de reunir un quórum calificado de dos terceras partes de los votos, para que así cuando llegue a reunirse dicho quórum, y más todavía cuando éstos aumenten, se tenga la seguridad, punto menos que completa, de que la reforma iniciada se recomienda por su justicia y su conveniencia y no deje duda de que representa verdaderamente la voluntad de la nación".

Ahora bien, resulta claro que el quórum de votación de $2 / 3$ partes que exige el artículo constitucional en comento, tendrá un alcance diferente si se exige respecto del total de los integrantes del Congreso de la Unión, o de las cámaras que lo componen, o si tan sólo se exige respecto de los miembros presentes en la sesión respectiva de dichos cuerpos colegiados. En el caso, la Constitución vigente exige que la propuesta de reforma 
constitucional sea aprobada por el voto de las $2 / 3$ partes de los integrantes del Congreso de la Unión que se encuentren presentes.

En vista de ello para saber si una propuesta de reforma a la Constitución fue aprobada por el quórum de votación requerido, se requiere, en primer término, conocer los términos en que se configuró en la sesión respectiva el quórum de asistencia que exige la Constitución, y el cual es, en ambas cámaras, de simple mayoría. Ahora bien, toda vez que la Cámara de Diputados se integra con 500 representantes, para que dicho cuerpo colegiado pueda sesionar y adoptar resoluciones, deben encontrarse presentes, al menos, 251 diputados, y en caso de que de esta forma se haya constituido el quórum de asistencia, entonces el quórum de votación consistente en $2 / 3$ partes de los miembros presentes, se colmará con 167 votos en favor de la reforma $(251 / 3=83 \times 2=167)$, lo que representa $1 / 3$ parte de los integrantes de la referida cámara. El mismo razonamiento se debe de hacer en relación con el Senado que se integra con 128 senadores, en cuyo caso el quórum de asistencia de simple mayoría se colmará con 65 senadores, por lo que el quórum de votación requerido, del voto favorable de $2 / 3$ partes de los miembros presentes, se satisfacerá con 43 votos en favor de la reforma en cuestión $(65 / 3=21.6 \times 2=43)$.

Con base en las consideraciones anteriores, se puede concluir que el propósito de que persigue la exigencia de un quórum de votación calificada que "no deje duda de que representa verdaderamente la voluntad de la nación", se verá frustrado si dicho quórum calificado se exige, no en función de los integrantes del cuerpo colegido en cuestión, sino en relación con la mayoría que se encuentre presente en la sesión, ya que en este caso se puede satisfacer el quórum de votación requerido con el voto de 167 de los 500 diputados y con el voto de 43 de los 128 senadores, lo que equivale a 1/3 parte de los integrantes del Congreso de la Unión, situación que se compagina mal con el principio básico de toda democracia consistente en la que las decisiones se deben tomar por mayoría y además está lejos de asegurar que la reforma en cuestión cuenta con un amplio consenso en favor de la misma.

Es por ello que he propuesto que se denomine "cláusula Copperfield", al quórum calificado de votación de 2/3 partes de los miembros del Congreso de la Unión cuando se le agrega la expresión "que estén presentes", ya que con dicha expresión se introduce un artificio que da como resultado que el quórum de votación calificado de 2/3 partes, se convierta, en la hipótesis 
planteada, en un quórum de votación real equivalente a 1/3 parte de los integrantes del Congreso.

Pero la aplicación de la fórmula empleada por la Constitución dará resultados diametralmente opuestos, si en la sesión en la que se va a conocer de la propuesta de reforma constitucional el quórum de asistencia se integra con la totalidad de los integrantes de la Cámara de Diputados, que son 500 , pues en tal caso la exigencia de que las propuestas de reformas a la Constitución tengan que ser aprobadas por el voto de las $2 / 3$ partes de los miembros presentes, exigirá el voto favorable de 333 diputados, y en tal hipótesis se le conferirá un poder extraordinario a la minoría contraria a la reforma, ya que bastará la oposición de 168 diputados, que representan 1/3 parte de los integrantes de la cámara, para frustrar la voluntad de 332, que representan 2/3 partes del referido cuerpo colegiado, situación que, como en el caso anterior, se compagina mal con los principios fundamentales de la democracia.

Con base en las consideraciones anteriores, se puede decir que en caso de que en la sesión de resolución de una propuesta de reforma constitucional el quórum de asistencia se configure con la simple mayoría de los integrantes del cuerpo colegiado en cuestión, el quórum calificado de votación exigido ( $2 / 3$ partes de los presentes), favorecerá a la minoría promovente de la reforma constitucional, en tanto que en la medida en que el quórum de asistencia se configure con un número mayor de integrantes y sobre todo en los casos en que se integre con la totalidad de los integrantes del referido cuerpo colegiado, el quórum de votación exigido (2/3 partes de los presentes, que en este caso lo serán todos o casi todos) favorecerá a la minoría opositora a la reforma en cuestión.

4. ¿Por qué "mayoría" deben de aprobar las legislaturas de los estados las propuestas de reforma a la Constitución federal que les sea remitidas después de que hayan sido aprobadas por el Congreso de la Unión?

El problema se plantea toda vez que la Constitución federal determina la forma y términos como se deben votar en el Congreso de la Unión las propuestas de reforma a la Constitución, pero no precisa la mayoría con la cual deben ser aprobadas en las legislaturas locales, ya que se limita a exigir que la propuesta de reformas a la Constitución federal, una vez aprobadas por las $2 / 3$ partes de los miembros presentes del Congreso de la Unión, deberá ser aprobado por la mayoría de las legislaturas, es decir, por al menos 17 de las 32 existentes, pero sin especificar el tipo de ma- 
yoría con la cual se debe aprobar la propuesta de reforma en el seno de cada una de ellas.

En vista de ello, todo hace pensar que este tipo de determinación debería ser objeto de atención en las Constituciones locales, pero el análisis de las mismas permite afirmar que dichas Constituciones prevén el tipo de mayoría con que se deben aprobar las propuestas de reforma a la Constitución local, pero no contienen disposición alguna respecto a la mayoría con la cual la legislatura estatal debe de aprobar las propuesta de reforma a la Constitución federal.

La falta de disposición al respecto en las Constituciones de los estados, ha dado como resultado que en algunos de ellos se resuelva, que el quórum de votación para aprobar una propuesta de reforma a la Constitución federal es de simple mayoría de los diputados integrantes de la legislatura que se encuentren presentes, lo que contrasta con el hecho de que en sus Constituciones locales se exija que las propuestas de reforma a la Constitución local respectiva deban ser aprobadas por el voto de las $2 / 3$ partes del total de los integrantes de la legislatura y no solo de los presentes.

A efecto de evitar que se presenten situaciones como la descrita, se considera conveniente el que las Constituciones locales exijan el mismo quórum de votación para aprobar las propuestas de reforma a la Constitución federal que a la local, ya que bien vistas las cosas, la Constitución federal y la local configuran, en su conjunto, la Constitución de la entidad.

5. De conformidad a la regulación vigente, no existe un término expreso o tácito para que las legislaturas de los Estados aprueben o rechacen una propuesta de reformas a la Constitucional federal que les haya sido remitida por el Congreso de la Unión o por conducto de su Comisión Permanente, lo que puede dar como resultado el que en los hechos esta práctica se convierta en un especie de veto de las legislaturas de los estados a las determinaciones del Congreso de la Unión

En vista de ello, se han formulado propuestas para que se señale en forma expresa en el artículo 135 constitucional, un plazo dentro del cual necesariamente se deben pronunciar las legislaturas locales, debiéndose precisar que en caso de que transcurra el plazo fijado si la legislatura en cuestión no hace ningún pronunciamiento, se deberá entender, por ese simple hecho, que aprueba el proyecto en cuestión (afirmativa ficta). Cabe precisar que el plazo que se fije al efecto debe ser suficiente (entre 15 y 45 días naturales) para que las legislaturas cuenten con tiempo para analizar con 
cuidado los proyectos de reforma a la Constitución federal que se sometan a su consideración.

6. ¿Tiene el poder constitucional reformador algún límite en su actuación? El poder constitucional reformador, ya sea que se conforme con apego al modelo central o federal ¿tiene algún límite en su actuación, o por el contrario, puede modificar cualquier disposición constitucional e incluso toda la Constitución?

Una primera corriente de tratadistas es del parecer de Felipe Tena Ramírez, que considera que el poder constitucional reformador no puede tener límite alguno en su actuación, ya que si se acepta la tesis de que dicho poder no puede modificar cualquier disposición constitucional, debe concluirse que para poder modificar la Constitución más allá del límite autorizado, se hará necesario salirse de los límites trazados por la propia Constitución, y en este caso se incurrirá en una aberración jurídica, ya que resulta absurdo que el derecho sancione una forma de violar al derecho. En consecuencia, para esta corriente el poder encargado de reformar a la Constitución no puede tener límite alguno en su actuación y menos aún si estos no se hacen constar expresamente en la Constitución.

Para otra corriente, que orbita en torno al pensamiento de Schmitt, el poder constitucional reformador puede ampliar, mejorar o perfeccionar cualquier precepto constitucional, incluso las decisiones políticas fundamentales contenidas en la misma, pero no puede suprimir, limitar o restringir ninguna de dichas decisiones, ya que a éstas, por su propia naturaleza, sólo el Poder Constituyente puede reformarlas. Esta postura plantea un doble problema ya que en primer término, se requiere determinar cuáles son las decisiones políticas fundamentales contenidas en cada Constitución en lo particular, así como la talla y los contornos de cada una de ellas y una vez hecho lo anterior desentrañar si la propuesta de reforma amplía o restringe a alguna decisión política fundamental.

El problema se agudiza debido a que toda decisión política fundamental se configura con un racimo de disposiciones contenidas en varios artículos, por lo que la reforma de cualquiera de ellos puede entrañar una modificación substancial a una decisión de este tipo. Así, si bien es cierto que el principio medular del sistema federal mexicano se encuentra contenido en el artículo 124, que se refiere al reparto de competencias entre la Federación y las entidades federativas, también lo es que los contornos fundamentales de dicho principio están trazados, por los artículos 73, 89 
y 94 constitucionales, ya que en ellos se precisan las atribuciones que la Constitución le confiere a los poderes de la Federación.

Las consideraciones anteriores han dado como resultado el que en los últimos años haya ganado terreno una tercera corriente que parte de la consideración de que si toda Constitución es producto de la voluntad general, lo que el pueblo ha decidido sólo el pueblo puede modificarlo. Esta corriente, considera que al poder constitucional reformador (ya sea que se configure con apego al modelo central, al federal, o a cualquier otro modelo) se le debe conferir una naturaleza proyectista y no determinativa, por lo que se debe disponer que todo proyecto de reforma constitucional que sea aprobado por el poder constitucional reformador para llegar a formar parte de la Constitución, requerirá, además, de la aprobación definitiva del pueblo por la vía del referéndum, como lo establecen las Constituciones de Francia y de los Estados Unidos.

Así, la Constitución Francesa de 1958 dispone en el artículo 89 que la iniciativa de reformas a la Constitución le corresponde al presidente de la República, a propuesta del Primer Ministro y a los miembros del Parlamento y también dispone que toda propuesta de reforma debe ser votada por las dos asambleas (Asamblea Nacional y Senado) en términos idénticos y será definitiva una vez que haya sido aprobada por el cuerpo ciudadano mediante referéndum. Ahora bien, la Constitución prevé que un proyecto de reformas a la misma no sea sometido a Referéndum en caso de que el presidente de la República decida someterlo al Parlamento convocado en Congreso, es decir, cuando la Asamblea Nacional y el Senado sesionan en forma conjunta en el Palacio de Versalles; en este caso, la propuesta de reformas para ser aprobada requiere de una mayoría de $3 / 5$ partes de los votos emitidos.

El artículo 5o. de la Constitución Federal de los Estados Unidos dispone que siempre que las $2 /{ }_{3}$ partes de ambas cámaras lo juzguen necesario, el Congreso propondrá enmiendas a la Constitución o bien a solicitud de dos tercios en los Estados, a cuyo efecto se convocará a una comisión para que proponga las enmiendas. Ahora bien, de conformidad con lo dispuesto por el referido artículo la propuesta de reforma en uno y otro caso pasará a formar parte de la Constitución, una vez que hayan sido ratificadas por las legislaturas de las $3 / 4$ partes de los Estados, separadamente o por medio de convenciones reunidas en $3 / 4$ partes de las mismas, según que el Congreso haya propuesto uno u otro modo de hacer la ratificación. 
Otros aspectos relevantes que por razones de espacio y tiempo en esta ocasión tan solo enunciaré son los siguientes:

7. ¿Puede el presidente de la República vetar una reforma a la Constitución federal aprobada en los términos y condiciones establecidos por la Constitución?

8. ¿Cual es el alcance real de aquellas disposiciones que algunos autores llaman "cláusulas pétreas", y otros "cláusulas intangibles" a través de las cuales se prohíbe modificar ciertas disposiciones, como puede ser la libertad de expresión, la religión de estado o el sistema federal?

9. ¿Puede el poder constitucional reformador aprobar una propuesta de reforma constitucional que implique su propia destrucción?

10. ¿Puede una reforma constitucional ser declarada inconstitucional, ya sea por no haberse observado escrupulosamente el procedimiento establecido para ello, o por cuanto su contenido choque con otras disposiciones constitucionales?

11. La exigencia contenida en el artículo 135 constitucional conforme a la cual toda propuesta de reformas a la Constitución federal requiere, entre otros requisito, de la aprobación de la mayoría de las legislaturas de los Estados, plantea el problema de determinar si la legislatura del Distrito Federal tiene voto en los procesos de reforma constitucional ya que si bien es una entidad federativa, no es un Estado y en caso de que la respuesta sea negativa, cabria preguntarse si al menos tiene derecho a participar en los procesos de reforma constitucional relacionados con su organización $\mathrm{y}$ funcionamiento.

12. ¿En qué términos se debe hacer del conocimiento de la ciudadanía el que una iniciativa de reformas a la Constitución federal ha sido aprobada en la forma y términos exigidos por la Ley Suprema? En esta materia se debe de tener presente que "la forma es fondo", y que el poder constitucional reformador no se confunde con los órganos que lo integran, ni con el órgano que hace la declaratoria de que una reforma ha sido aprobada con apego al procedimiento establecido por la Constitución.

Con base en las consideraciones anteriores se puede decir que conclusión el reconocimiento del referéndum en términos eficientes en los procesos de reforma constitucional, requiere como condición previa el que se mejoren los mecanismos de reforma constitucional vigentes. 